\title{
Introduction to the Special Issue on the Award Papers of USENIX ATC 2019
}

This special issue of ACM Transactions on Computer Systems presents the three papers from the 2019 USENIX Annual Technical Conference (ATC'19) that won the Best Paper Award. The scope of ATC is broad. It covers all practical aspects related to systems software, and its goal is to improve and further the knowledge of computing systems of all scales, from small embedded devices to large data centers, while emphasizing implementations and experimental results. ATC underwent significant changes and improvements in $2019 .^{1}$

ATC'19 received 356 submissions and accepted 71 (nearly 20\%) through a double-blind, tworound review process in which each round 2 submission was reviewed by 5 to 6 program committee (PC) members. After the ATC'19 program was finalized, the Best Paper Award selection process proceeded in two phases. In the first phase, we combined several signals. One was an explicit ranking by reviewers marking papers worthy of consideration for the best paper, and any paper marked for such consideration by two or more PC members was passed to the second phase. Additionally, we considered general review ranks and deliberations (both online and during the PC meeting), moving several additional top-ranking papers to the second phase. Last, we collected explicit nominations by PC members for the Best Paper Award.

At the end of the first phase, we generated a short-list of eight candidate papers. At this stage, we appointed a team of six PC members consisting of senior and experienced members of the systems research community. During a period of 4 weeks, the team read the papers, and we discussed each separately for best paper worthiness. We did not place a quota on the number of Best Paper Awards. Generally, the committee favored papers with original or surprising contributions, and/or ones that would spark interest and establish a new direction for follow-on works. At the end of the second stage, we elected three papers to receive the ATC'19 Best Paper Award, which are presented in this special issue. All three works include additional material relative to their conference version, which has been reviewed (in "fast-track mode") by one or two of the original ATC'19 reviewers.

The first work is "SILK+: Preventing Latency Spikes in Log-Structured Merge Key-Value Stores Running Heterogeneous Workloads” by Oana Balmau, Florin Dinu, Willy Zwaenepoel, Karan Gupta, Ravishankar Chandhiramoorthi, and Diego Didona, which introduces techniques that manage to lower the 99th percentile latencies by up to two orders of magnitude relative to common log-structured merge key-value stores. The second work is "Transactuations: Where Transactions Meet the Physical World” by Aritra Sengupta, Tanakorn Leesatapornwongsa, Masoud Saeida Ardekani, and Cesar A. Stuardo, which uncovers IoT application inconsistencies manifesting out of failures and proposes a useful abstraction and execution model to combat this problem.

\footnotetext{
${ }^{1}$ D. Malkhi and D. Tsafrir. 2019. Refreshing ATC. In ATC'19: USENIX Annual Technical Conference. xi-xxviii. https://www. usenix.org/sites/default/files/atc19-message.pdf.
}

\footnotetext{
ACM Reference format:

https://doi.org/10.1145/3395034

(C) 2020 Copyright held by the owner/author(s).

0734-2071/2020/05-ART11

https://doi.org/10.1145/3395034
}

Dahlia Malkhi and Dan Tsafrir. 2020. Introduction to the Special Issue on the Award Papers of USENIX ATC 2019. ACM Trans. Comput. Syst. 36, 4, Article 11 (May 2020), 2 pages. 
The third work is “A Retargetable System-Level DBT Hypervisor” by Tom Spink, Harry Wagstaff, and Björn Franke, which develops a dynamic binary translation (DBT) hypervisor generated using high-level guest machine specifications and simultaneously simplifies retargeting of the DBT to new architectures while delivering performance levels in excess of existing manually optimized DBT solutions. It is our hope that you will find these works interesting and insightful.

The ATC'19 program is the result of tremendous efforts by many in the systems community. We are most thankful to the authors who submitted their high-quality work and to the reviewers who undertook the challenging task of evaluating hundreds of submissions and providing constructive feedback to the authors.

Dahlia Malkhi

Dan Tsafrir

Program Co-Chairs

Guest Editors 